\title{
eNeuro
}

Research Article: New Research / Cognition and Behavior

\section{Mediodorsal Thalamus Is Critical for Updating during Extradimensional Shifts But Not Reversals in the Attentional Set-Shifting Task}

https://doi.org/10.1523/ENEURO.0162-21.2022

Cite as: eNeuro 2022; 10.1523/ENEURO.0162-21.2022

Received: 14 April 2021

Revised: 5 January 2022

Accepted: 11 January 2022

This Early Release article has been peer-reviewed and accepted, but has not been through the composition and copyediting processes. The final version may differ slightly in style or formatting and will contain links to any extended data.

Alerts: Sign up at www.eneuro.org/alerts to receive customized email alerts when the fully formatted version of this article is published.

Copyright (@ 2022 Ouhaz et al.

This is an open-access article distributed under the terms of the Creative Commons Attribution 4.0 International license, which permits unrestricted use, distribution and reproduction in any medium provided that the original work is properly attributed. 
1 Full title: Mediodorsal thalamus is critical for updating during extra-dimensional shifts but

2 not reversals in the attentional set-shifting task

3

4 Abbreviated title: Mediodorsal thalamus and choice response strategy updating

5

6 Authors: Zakaria Ouhaz ${ }^{1,2}$, Brook AL Perry ${ }^{1}$, Kouichi Nakamura ${ }^{3}$, Anna S Mitchell ${ }^{1}$

81 - Department of Experimental Psychology, University of Oxford, The Tinsley Building,

9 Mansfield Road, Oxford OX1 3SR, United Kingdom

102 - Institut Supérieur des Professions Infirmières et Techniques de la Santé, Marrakech,

11 Morocco

123 - Medical Research Council Brain Network Dynamics Unit, Nuffield Department of

13 Clinical Neurosciences, University of Oxford, Mansfield Road, OX1 3TH, United Kingdom

Author contributions: $\mathrm{ZO}$ and ASM designed research; ZO performed research; $\mathrm{KN}$ and

BALP contributed unpublished reagents/ analytic tools; ZO, BALP, ASM analyzed data; ZO, BALP, KN, ASM wrote the paper.

Correspondence should be addressed to ASM. anna.mitchell@psy.ox.ac.uk

Number of Figures: 4 and a figure in Extended Data Figure 2-1

Number of Tables: 1

Number of words for Abstract: 146 words

Number of words for Significance: 120 words

Number of words for Introduction: 769 words

Number of words for Discussion: 3178 words

Acknowledgements

We are very grateful for support received from Professor Verity Brown and Dr David Tait, who taught $\mathrm{ZO}$ to perform the ID/ED attentional set-shifting task during an extended visit to their research lab at St Andrews University.

Funding sources: ZO was supported by a Royal Society Newton International Fellowship, NF160862. ASM was funded by the Wellcome Trust [Grant number WT110157/Z/15/Z]. For the purpose of open access, the author has applied a CC BY public copyright to any Author Accepted Manuscript version arising from this submission.

Authors report no conflict of interest 
40 Abstract

41 Cognitive flexibility, attributed to frontal cortex, is vital for navigating the complexities of

42 everyday life. The mediodorsal thalamus (MD), interconnected to frontal cortex, may

43 influence cognitive flexibility. Here, male rats performed an attentional set-shifting task

44 measuring intra-dimensional and extra-dimensional shifts in sensory discriminations. MD

45 lesion rats needed more trials to learn the rewarded sensory dimension. However, once the

46 choice response strategy was established, learning further two-choice discriminations in the

47 same sensory dimension, and reversals of the reward contingencies in the same dimension,

48 were unimpaired. Critically though, MD lesion rats were impaired during the extra-

49 dimensional shift, when they must rapidly update the optimal choice response strategy.

50 Behavioral analyses showed MD lesion rats had significantly reduced correct within-trial

51 second choice responses. This evidence shows that transfer of information via the MD is

52 critical when rapid within-trial updates in established choice response strategies are required

53 after a rule change.

\section{Significance statement}

56 We demonstrate for the first time that rodent mediodorsal (MD) thalamus is a critical node

57 when choice response strategies need to change rapidly after a within-session rule change

58 during reward-guided learning. MD interactions with orbitofrontal cortex are critical for

59 value-based learning, while MD interactions with medial prefrontal cortex are critical for

60 rapid within-trial updating of optimal choice response rules. MD interactions with the

61 orbitofrontal cortex are not always necessary for reversal learning. These results indicate that

62 the MD also contributes to attentional set-shifting. Our evidence advocates for studies

63 investigating deficits in updating optimal choice response strategies due to potentially

64 disrupted cortico-thalamocortical transfer of information in people diagnosed with 
65 Alzheimer's disease combined with more frontal pathology, Parkinson's disease, or 66 schizophrenia.

67 
69 Cognitive flexibility describes our ability to quickly and selectively switch our thoughts, responses, or behavior to everyday dynamic situations. This capacity to rapidly update or

71 alter one's actions conveys evolutionary benefit and is key to survival (Koechlin et al., 2003;

72 Rougier et al., 2005). Cognitive flexibility shows marked changes or declines in

73 neurodegenerative disorders, like Parkinson's disease, in patients with ventral prefrontal cortex (PFC) damage, and in neurodevelopmental diseases, like schizophrenia (Andrews et al., 2006; Barch et al., 2009; Clark et al., 2004; Giraldo-Chica et al., 2018).

Cognitive flexibility is not unique to primates. Studies show that rats can readily switch between attentional sets to optimise reward outcome (Birrell and Brown, 2000; McAlonan and Brown, 2003; Newman and McGaughy, 2011). Typically, these studies have focussed on various frontal cortex subregions. Yet, it is becoming increasingly clear that the cortex does not function in isolation, but rather, relies heavily on subcortical and peripheral inputs supplied by the thalamus (Sherman and Guillery, 2002; Guillery and Sherman, 2011; Halassa and Kastner, 2017). The mediodorsal thalamus (MD) has a critical role in functions of frontal cortex during higher order cognitive processes across mammalian species (Alcaraz et al., 2018; Browning et al., 2015; Chakraborty et al., 2016; Chakraborty et al., 2019; DeNicola et al., 2020; Ferguson and Gao, 2018; Miller et al., 2017; Mitchell and DalrympleAlford, 2005; Mitchell et al., 2007a,b; Mitchell and Gaffan, 2008; Parnaudeau et al., 2013; Pergola et al., 2018; Perry et al., 2021; Schmitt et al., 2017). This evidence highlights that the MD and PFC are working in partnership, contributing different but complementary roles to goal-directed behaviors (Mitchell, 2015; Perry et al., 2021).

In mammals, the MD is densely interconnected with areas of frontal cortex involved

91 in various aspects of cognitive flexibility. Recent work shows MD projections to frontal

92 cortex allow for the simultaneous sharing of information across multiple cortical regions. 
93 This distributed pattern of frontal innervation by the MD appears to be especially true for the 94 central (MDc) and medial (MDm) subdivisions, which have projections to the orbitofrontal 95 cortex (OFC), medial PFC, and frontal association areas (Alcaraz et al., 2016; Dajani and

96 Uddin, 2015; Groenwegen, 1988; Kuramoto et al., 2017; Perry et al., 2021). Although

97 phylogenetic differences exist in the organisation of rodent and primate frontal cortex

98 (Preuss, 1995), similar functional contributions to aspects of cognitive flexibility are

99 observed in monkeys and rodents after perturbations to similar areas of agranular frontal

100 cortex (Birrell and Brown, 2000; Bissonette et al., 2008; Dias et al., 1996; Dias et al., 1997;

101 McAlonan and Brown, 2003). Given there are similarities in neuroanatomical connectivity

102 between agranular frontal cortex and MD in rodents and primates, it seems that cognitive

103 flexibility and rapid updating deficits observed in monkeys with excitotoxic MD lesions

104 (Browning et al., 2015; Chakraborty et al., 2016; Mitchell et al., 2007a; Mitchell and Gaffan,

105 2008) may also extend to rodents.

106 Thus, in the current study, rats with excitotoxic lesions to the MD or MD Sham

107 controls were run on a well-established test of cognitive flexibility - the intra-dimensional

108 (ID)/ extra-dimensional (ED) attentional set-shifting task (Birrell and Brown, 2000). To test

109 this premise and investigate any deficits in choice responding, we used the standard 7-stage

110 version comprising of multiple subtasks all conducted within a single testing session (Birrell

111 and Brown, 2000; Chase et al., 2012). In rats, the ID/ED task has produced dissociable

112 deficits after permanent cortical or limbic thalamic lesions, or neuropharmacological

113 manipulations (e.g., Linley et al., 2016; McGaughy et al., 2008; Tait et al., 2014; Tait et al.,

114 2018; Wright et al., 2015).

115 In the current experiment, we predicted MD lesion rats would show deficits during

116 the initial sensory acquisition (simple discrimination, SD) and the ED shift. Both the SD and

117 ED subtasks require rapid, within trial learning or updating of choice response strategies. 
118 Therefore, impaired SD and ED performance would be consistent with the selective learning

119 deficits observed in other rodent studies after MD perturbations (Chakraborty and Mitchell,

120 2013, for review; Courtiol et al., 2019; Ferguson et al., 2017; Hunt and Aggleton, 1998;

121 Mitchell and Dalrymple-Alford, 2005; Perry et al., 2021). In addition, we predicted our MD

122 lesion rats may show deficits during reversal subtasks. MD lesions in rodents can produce

123 reversal learning deficits, although the evidence is mixed (see Chakraborty and Mitchell,

1242013 for review). However, given the reciprocal direct MD connectivity with the OFC

125 (Groenwegen, 1988; Krettek and Price, 1977; Ray and Price, 1992), and the selective deficits

126 observed in rodents and monkeys with OFC manipulations performing the reversal subtasks

127 during the ID/ED attentional set-shifting task (Dias et al., 1996, McAlonan and Brown,

128 2003), it remained an open question.

\section{Materials and Methods}

131 Animals: Twenty-five Lister hooded male rats weighing between 420 and $480 \mathrm{gm}$ at time of 132 surgery (Charles River, UK) were group housed in a temperature and humidity-controlled 133 environment $\left(21 \pm 1^{\circ} \mathrm{C}\right)$. The housing and husbandry compiled with the ARRIVE guidelines 134 of the European Directive (2010/63/EU) for the care and use of laboratory animals. Testing 135 was conducted in the light phase of a $12 \mathrm{hr}$ light/dark cycle (lights on at $7 \mathrm{am}$ ). The rats were 136 maintained on a controlled feeding schedule ( $20 \mathrm{~g} / \mathrm{rat} / \mathrm{day})$ with water freely available in the 137 home cage. All experimental procedures were performed in compliance with the United 138 Kingdom Animals (Scientific Procedures) Act of 1986. A project license authorized all 139 procedures after review by the university animal care and ethical review committee and 140 Home Office Inspectorate.

141 Surgery: Rats were anesthetized by isoflurane and oxygen mix (4\% induction, $1.8-2 \%$

142 maintenance), and given analgesia in subcutaneous injections of $0.05 \mathrm{mg} / \mathrm{kg}$ buprenorphine 
143 (Vetergesic; $0.3 \mathrm{mg} / \mathrm{ml}$ ) and $1 \mathrm{mg} / \mathrm{kg}$ Metacam (Meloxicam; $5 \mathrm{mg} / \mathrm{ml}$ ). Rats were secured in

144 a stereotaxic frame (Kopf) with atraumatic ear bars and the nose bar set to $+3.3 \mathrm{~mm}$ to

145 achieve a level skull. A subcutaneous injection of $2 \mathrm{mg} / \mathrm{kg}$ bupivacaine (Marcaine; 2.5

$146 \mathrm{mg} / \mathrm{ml}$ ) was administered into the scalp in the location of the midline incision. Viscotears was

147 applied to keep the eyes moist. During surgery, each rat was placed on a heat pad and

148 covered in bubble wrap with an internal rectal thermometer probe to monitor and maintain

149 normal body temperature. Warmed sterile saline $(1 \mathrm{ml} / 100 \mathrm{gm})$ was administered

150 subcutaneous into the scruff of the neck after one hour. A midline incision was performed.

151 Bregma and lambda coordinates were determined. A dental drill with a trephine head was

152 used to create a craniotomy over the midline. To maximize lesion accuracy, anterior and

153 posterior injection sites were calculated according to the bregma-lambda distance in each rat.

154 Coordinates were for anterior MD injection: anterior-posterior $(\mathrm{AP})=-0.395 \mathrm{~mm}$, medial-

155 lateral $(\mathrm{ML})=-0.1$ (avoiding the superior sagittal sinus, which was visible within the dura

156 inside the craniotomy) and dorsal-ventral (DV) $=0.56 \mathrm{~mm}$ (from dura), volume of

157 excitotoxin $=0.20 \mu \mathrm{l}$; for posterior $\mathrm{MD}: \mathrm{AP}=-0.435 \mathrm{~mm}, \mathrm{ML}=+0.1$ and $\mathrm{DV}=0.57 \mathrm{~mm}$

158 (from dura), volume of excitotoxin $=0.18 \mu 1$. Fifteen rats (MD lesion) received $0.12 \mathrm{M}$ of

159 NMDA (N-methyl-D-Aspartate) dissolved in phosphate buffer (pH 7.20) in each hemisphere

160 from a $1 \mu 1$-gauge Hamilton bevelled-tip syringe. The injections were performed manually,

161 taking 3 min each, and after injection the needle was left in situ for a further 3 min to allow

162 diffusion and to limit wicking of the NMDA. A further 10 rats (MD Sham controls) received

163 injections of sterile phosphate buffer instead of NMDA using the same injection coordinates.

164 Injections were given bilaterally in the MD. Upon completion of surgery, wounds were

165 sutured with Vicryl 4.0. Rats were housed singly during recovery for up to three days and

166 were then returned to their pre-surgery housing cohort. Postoperative oral analgesia, $1 \mathrm{mg} / \mathrm{kg}$

167 Metacam (Meloxicam, $1.5 \mathrm{mg} / \mathrm{ml}$ ) dissolved and set in jelly was provided in individual bowls 
to each rat daily for three days. Behavioral and physiological evidence showed that all rats recovered well, with normal eating and pre-surgery weights returning within $24 \mathrm{~h}$. Food

170 regulation $(20 \mathrm{gm} /$ day/rat $)$ started again $10 \mathrm{~d}$ after recovery. Postoperative testing began at

171 least $15 \mathrm{~d}$ after surgery. The researcher was blind to the lesion group of each animal until all

172 tests were completed.

\section{ID/ED Attentional Set Shifting paradigm}

174 Apparatus: The test chamber consisted of a modified Plexiglass home cage $(40 \times 70 \times 18$

$175 \mathrm{~cm})$ with Perpex dividers separating the cage into a large start chamber $(40 \times 46 \mathrm{~cm})$ and two

176 identically sized $(24 \times 20 \mathrm{~cm})$ choice chambers to which access was controlled by removable

177 Perspex doors. The digging bowls were ceramic (internal diameter $7 \mathrm{~cm}$, depth $4 \mathrm{~cm}$ ) and

178 were placed within the choice chambers. The bowls were filled with digging media of

179 different textures, and the digging media were scented with different spices (see Table $\mathbf{1}$ for

180 examples). The odor or digging media discriminations, pairs of stimuli used, and the correct

181 stimulus within pairs were counter-balanced across subtasks and matched between groups.

182 The bowls were baited with Honey Nut Cheerios (Nestle®), each bowl contained a hidden

183 (securely fixed under a metal grid) Honey Nut Cheerio to serve as an odor mask. During

184 testing, only one of the bowls was baited and rats determined which bowl was baited using

185 either the texture of the digging medium or the odor as cues. Prior to testing, rats were taught

186 to dig in bowls filled with home-cage bedding material to retrieve one half of a Honey Nut

187 Cheerio. The task was performed as previously described in Birrell and Brown (2000) and

188 divided into two phases administered on two consecutive days.

189 Preoperative and Postoperative Training Day: On the day before testing, each rat learnt

190 one simple two-choice discrimination (SD) using either of the two sensory dimensions: odor

191 (mint vs oregano), or digging media (shredded paper vs cotton pads), to a criterion of six 
192 consecutive correct trials. The rewarded odors or digging media were counterbalanced across

193 the two groups, and these exemplars were not used again throughout testing. Digging was

194 defined as active digging with both front paws or active foraging with the snout in the

195 digging media. Sniffing or touching the media with the paws was not scored as a dig.

Preoperative and Postoperative Testing paradigm: The following day, each rat was given a series of seven two-choice discriminations (subtasks) in the following order: a new simple discrimination (SD) using either of two sensory dimension (odor or digging media); a compound discrimination $(\mathrm{CD})$ using the same rewarded sensory dimension and two-choice discrimination as the SD, combined with the other irrelevant sensory dimension; first reversal (REV1), in which the exemplars remained the same as in the CD but the correct (rewarded) and incorrect exemplars were reversed; intra-dimensional shift (ID), in which new exemplars were used, but the relevant sensory dimension remained the same as in the previous subtasks; second reversal (REV2), where exemplars in the ID remained the same but the reward contingencies of the two exemplars were reversed; extra-dimensional shift (ED), where new exemplars were used and the previously irrelevant sensory dimension becomes relevant; and a third reversal (REV3), where exemplars in the ED subtask remained the same but the reward contingencies of the two exemplars were reversed.

The task is self-paced and relies on the natural foraging behaviors of the rats. The first 210 five two-choice discrimination subtasks (SD, CD, REV1, ID, REV2) must be solved by 211 discriminating between exemplars from the same sensory dimension. In this stage of the task,

212 the rat is rewarded for choices based on specific perceptual features of the stimuli, while

213 ignoring other features that also distinguish the stimuli. After acquiring each set of two-

214 choice discriminations to a performance criterion of six correct consecutive trials, the rats 215 encounter a reversal of the reward contingencies associated with the exemplars (a reversal 216 subtask: REV1 and REV2). For the final two subtasks (ED and REV3), exemplars from the 
217 previously irrelevant sensory dimension become relevant. Therefore the rat must shift its

218 attention (and adjust its choice response strategy) to the previously irrelevant sensory

219 dimension and perceptual feature to receive reward (ED subtask). The attentional set-shfiting

220 cost is calculated by comparing trials to criterion during the intra-dimensional shift, where

221 the sensory dimension is consistent with previous subtasks, and the extra-dimensional shift,

222 where the now relevant sensory dimension had been previously ignored and thus requires a

223 shift to optimise continuing to retrieve rewards.

224 Odor detection test: At the completion of ID/ ED testing, the olfactory abilities of the rat to

225 smell the odor of the buried half-Honey Nut Cheerio were assessed. This task consisted of 10

226 consecutive trials where the rat was exposed to bowls containing similar bedding. The rat was

227 placed in the waiting area of the set-shifting apparatus. Similar bedding-filled bowls were

228 placed, one in each of the choice chambers, only one bowl contained half a Honey Nut

229 Cheerio at the bottom (pseudorandomly assigned to avoid the rat developing a response

230 strategy). The barrier was raised, allowing the rat access to both bowls. The rat had to chose

231 to dig into one bowl and had up to 15 minutes to make a digging response on each trial. If the

232 rat dug in the correct bowl, the trial was recorded as correct and the rat was returned to the

233 waiting area and the barrier lowered to block access to both choice chambers. If the rat dug in

234 the incorrect bowl, the trial was marked as incorrect. The rat was permitted to continue to

235 explore the incorrect bowl; the trial was terminated when the rat returned to the waiting area.

236 In all cases, the ability of each rat to detect the reward location was no different to chance

237 guessing.

238 Perfusion and histology: At the end of all experimental testing, rats were deeply

239 anaesthetized with sodium pentobarbital (Euthanol $1.0 \mathrm{ml} / \mathrm{rat}, 200 \mathrm{mg} / \mathrm{ml}$ : Merial, Harlow,

240 UK), and perfused transcardially with $0.9 \%(\mathrm{w} / \mathrm{v})$ saline and $4 \%(\mathrm{w} / \mathrm{v})$ paraformaldehyde in

$2410.1 \mathrm{M}$ phosphate buffered saline (PBS). Brains were then removed and post-fixed overnight 
$242\left(4^{\circ} \mathrm{C}\right)$, then incubated using $30 \%(\mathrm{w} / \mathrm{v})$ sucrose in $0.1 \mathrm{M}$ PBS over $48 \mathrm{~h}\left(4^{\circ} \mathrm{C}\right)$. Free floating

243 sections $(40 \mu \mathrm{m})$ were cut on a freezing microtome, the slices were then preserved in

244 cryoprotectant (0.1M PBS containing 24\% (v/v) glycerol and 24\% (v/v) ethylene glycol) and

245 stored at $-20^{\circ} \mathrm{C}$ until required. MD thalamus lesion locations were histologically verified

246 from cresyl violet stained brain sections.

247 Lesion extent: Photomicrographs of the Nissl-stained MD sections were captured using a

248 camera mounted on a freestanding Leica DMR microscope (Leica Microsystems) using a

249 1.6× objective so that the whole section could be visualised in the photomicrograph. MD

250 volumes were measured using the NIH software ImageJ (http://rsbweb.nih.gov/ij/). In each

251 rat, the intact MD volume from both the left and the right hemisphere was assessed. The final

252 reading was calculated according to the Cavalieri principle (Regeur and Pakkenberg, 1989)

253 and expressed as the percentage of MD Sham controls.

254 Behavioral data analysis: In the behavioral experiments, all rats performed a preoperative

255 and postoperative session, and they completed the seven two-choice discrimination subtasks

256 in an identical order. Data are expressed as mean and standard deviation. Preoperative and

257 postoperative mean trials to criterion, shift cost, percent correct $2^{\text {nd }}$ choice within trial

258 responses, and latency to dig (seconds) were analysed using repeated measures ANOVAs

259 with Stage (comprising the 7 subtasks) as the within-group repeated measure and

260 Lesion_type (MD lesion or MD Sham control) as the between-group factor. For significant

261 interactions, posthoc simple main effects analyses were performed using additional two-way

262 ANOVAs for repeated measures (e.g., ID/ED shift) or independent t-tests for relevant

263 subtasks (corrected for multiple comparisons). Univariate ANOVA was used to determine if

264 the Shift between the two sensory dimensions between the MD lesion or MD Sham control 
had an effect on the number of errors performed in the ED subtask. All statistical analyses were calculated using SPSS 24 software. The significance level was set at $p<0.05$.

268 Results

\section{MD lesion site}

270 Of the 25 adult rats involved in this experiment, 15 sustained MD lesions and 10 were MD 271 Sham controls (see Materials and Methods for details). All 15 MD lesion rats had extensive 272 excitotoxic (NMDA) lesion damage (see Fig. 1) within the medial and central subdivisions of 273 the MD, as intended. In all cases the lesion affected between $80-90 \%$ of the central and 274 medial MD, sparing only the lateral portions. The MD was consistently shrunken, and at the 275 lesion site there was significant cell loss. In all cases, the adjacent anterior thalamic nuclei 276 (ATN) were spared. In two cases, there was unilateral (right-sided) damage in the lateral habenulae $(\mathrm{LHb})$ and some damage to the central medial nucleus lying underneath the MD.

Include Figure 1 here

\section{The MD and attentional set-shifting}

282 Preoperatively, rats were pseudo-randomly assigned to either of two groups: MD lesion or 283 MD Sham control, based on the number of trials needed to reach the learning criterion (six correct consecutive trials in nine trials) during the simple discrimination (SD) subtask.

285 Preoperative data from one MD Sham control rat had to be discarded. An independent 286 samples t-test confirmed there was no significant difference between the two groups during 287 preoperative learning of the $\mathrm{SD}, t(22)=0.095, p>0.05$, with rats from both groups requiring

288 a similar number of trials to reach criterion, MD lesion $($ Mean $=9.67$, S.D. $=2.44)$ and MD 
Sham control $($ Mean $=9.78$, S.D. $=3.31)$. Overall trials to criterion during the preoperative training session are presented in Fig. $2 \mathrm{~A}$. ID, REV2, ED, REV3) as the within-group factor and Lesion_type (MD lesion vs MD Sham control) as the between-group factor revealed a significant interaction, Stage $\times$ Lesion_type, $F(6,138)=63.65, p<0.001$, a significant main effect of Stage, $F(6,138)=184.73, p<0.001$, and a significant main effect of Lesion_type, $F(1,23)=20.22, p<0.001$ (see Fig. 2B). To explore the interaction effect, posthoc comparisons of the simple main effects of Stage (corrected for multiple comparisons, $p<0.007$; alpha $=0.05$ divided by 7 tests) were computed. For the SD subtask, $\mathrm{MD}$ lesion rats required more trials to criterion $(\mathrm{M}=16.67$, S.D. = 1.23) compared with MD Sham controls $(M=9.50 ;$ S.D. $=2.07)$, indicating that the rats with the MD lesion were slower to acquire the new sensory discrimination. An independent samples t-test confirmed this difference was significant, $t(23)=10.88, p<0.001$. However, once the rats with MD lesion acquired the rewarded sensory dimension (to respond either to the digging media, or to the odor), they were unimpaired in subsequent subtasks that maintained the same rewarded sensory dimension, namely concurrent discrimination (CD): $t(23)=0.00, p=1.0$, and intra-dimensional shift (ID): $t(23)=1.08, p<0.291$. MD lesion rats were also not impaired in the reversals (REV1 and REV2) of the reward contingencies associated with the learned exemplars completed after the CD subtask, REV1: $t(23)=2.31, p$ $<0.030$, or after the ID subtask, REV2: $t(23)=1.34, p<0.192$, although, all rats were familiar with the reversal rule as they had completed the ID/ED task preoperatively (see

310 Materials and Methods). Extended Data Figure 2-1 shows the mean and $1^{\text {st }}$ and $3^{\text {rd }}$ quartile 311 box plots with the smallest and largest numbers of postoperative trials to criterion (whiskers) 312 for the individual rats during each of the subtasks. 
For the extra-dimensional (ED) subtask, the rats had to shift their attention to the

317 previously irrelevant (unrewarded) sensory dimension. Thus, given the rats had acquired the

318 attentional set strategy, as expected, all rats required more trials to criterion to learn the ED

319 subtask. MD Sham controls required $(M=15.70 ;$ S.D. $=1.64)$ more trials to learn the ED

320 when compared to trials required to learn the ID $(M=12.5$; S.D. $=1.84)$. Similarly, the MD

321 lesion rats required more trials $(M=23.47$, S.D. $=2.07)$ compared with trials to learn the ID

$322(\mathrm{M}=11.73 ;$ S.D. $=1.67$; see Fig. 2B $)$. A repeated measures ANOVA computing the trials to

323 criterion for these two subtasks (ID vs ED) showed a significant Stage x Lesion_type

324 interaction, $F(1,23)=99.96, p<0.001$, a significant effect of Stage, $F(1,23)=306.11, p$

$325<0.001$, and a significant effect of Lesion_type, $F(1,23)=32.83, p<0.001$ (see Fig. 2B). The

326 interaction occurred as the MD lesion rats required significantly more trials to criterion to

327 consistently implement a new choice strategy in order to learn which of two stimuli was

328 rewarded from the previously ignored sensory dimension compared to the MD Sham

329 controls, $t(23)=10.00, p<0.001$.

330 Interestingly, for REV3, the reversal subtask that occurred after the ED shift, MD

331 lesion rats required fewer trials to criterion $(M=12.13$, S.D. $=1.25)$ compared with the MD

332 Sham controls $(\mathrm{M}=16.7$; S.D. = 2.26; see Fig. 2B $)$. An independent samples t-test confirmed

333 this difference was significant, $t(23)=6.51, p<0.001$, suggesting a facilitation in

334 performance as a consequence of over-training experienced during the ED subtask (see

335 discussion for interpretation).

The additional repeated measures ANOVA of Session (Preoperative vs Postoperative)

337 shift cost (see Fig. 3A) between Lesion_type computed as the number of trials to criterion for

338 the ED subtask minus the number of trials to criterion for the ID subtask for each session 
showed a significant Session $\mathrm{x}$ Lesion_type interaction, $F(1,22)=44.06, p<0.001$, a significant effect of Sesion, $F(1,22)=38.27, p<0.001$, and a significant effect of

341 Lesion_type, $F(1,22)=15.46, p=0.001$ (see Fig. 3A). The significant interaction occurred as

342 there was a small shift cost for both groups during the preoperative test session. However,

343 during the postoperative test session only the MD lesion rats showed a significant shift cost

344 as a consequence of the task demands changing that required an adjustment to the previously 345 well-established response strategy $(p<0.001)$. By contrast, the MD Sham controls showed a 346 similar shift cost to their preoperative performance.

Insert Figure 3 here

\section{Within trial choice responses as a measure of rapid adaptability after MD lesion}

Given the observed deficits in the MD lesion group, we investigated the behavioral responses made in the MD lesion or MD Sham control rats. Given the task design and a criterion of six consecutive correct responses before switching to the next stage, overall during testing, the rats do not complete many trials. Nevertheless, we could determined whether the rats rapidly adapted their choice responses in the here and now (within trial choice responses) as measured by the number of correct $2^{\text {nd }}$ choice within trial responses made, i.e. when the rat dug from the correct bowl only after visiting the incorrect bowl first but without digging in it,

358 divided by the total number of correct responses (correct $1^{\text {st }}$ and $2^{\text {nd }}$ choice responses

359 combined) for each session. During each subtask of the session, we recorded if the rat made a 360 correct choice, either on the first attempt (' 1 st $c h o i c e$ response'), or on the $2^{\text {nd }}$ attempt (' 2 nd

361 choice within trial response'). We did not include error trials in this analysis. 
A repeated measures ANOVA of total percent correct $2^{\text {nd }}$ choice within trial

responses was conducted with Session (Preoperative vs Postoperative) as the repeated measure $\times$ Lesion_type revealed a significant interaction of Sesssion $\times$ Lesion_type, $F(1,21)$ $=9.40, p=0.006$, a significant main effect of Session, $F(1,21)=57.06, p<0.001$, and a

significant main effect of Lesion_type, $F(1,21)=7.18, p=0.014$ (see Fig. 3B). To

investigate the interaction effect, posthoc comparisons of the simple main effects showed that the $\mathrm{MD}$ lesion rats $(\mathrm{M}=28.18, \mathrm{SD}=7.61)$ made fewer correct $2^{\text {nd }}$ choice within trial responses compared to the MD Sham controls $(\mathrm{M}=35.23, \mathrm{SD}=8.46)$. This deficit suggests the MD lesion rats had a diminished ability to rapidly bind together their previous choice about what stimuli are worth sampling in order to update their choice response strategy within the trial, rather than a deficit in perseverative responding, or an inability to respond to negative feedback.

In addition, we analyzed the rats' errors made reaching criterion during the ED

subtask, as a consequence of whether they experienced a sensory dimension shift from odor to digging medium or digging medium to odor, which indicated that the increase in errors occurs irrespective of the direction of the attentional shift. The univariate ANOVA with Lesion_type and Sensory Shift as the between-subject factors and errors to criterion for the ED subtask as the dependent measure revealed a main effect of Lesion_type, $F(1,21)=$ 106.03, $p<0.001$, but no main effect of Sensory Shift, $F(1,21)=3.84, p=0.063$ and no interaction effect, $F(1,21)=0.19, p=0.666$.

\section{Latency to dig changes}

Latency to dig (in seconds) for error trials and correct trials were also computed for each rat in preoperative (see Fig. 4A; 4C) and postoperative (see Fig. 4B; 4D) analyses. Separate repeated measures ANOVAs were used to assess the latency to dig for the preoperative or 
postoperative sessions for either error trials or correct trials with Stage (7 subtasks: SD, CD, REV1 ID, REV2, ED, REV3) as the within-group factor and Lesion-type (MD lesion vs MD Sham control) as the between-group factor.

For error trials (Fig 4C), the repeated measures ANOVA for the preoperative session revealed a significant main effect of Stage, $F(1,21)=23.99, p<0.001$, but no effect of lesion, and no interaction, $F$ 's $<1.0$. This result indicates there was no difference in response times between the pseudo-randomly assigned groups (MD lesion and MD Sham controls) preoperatively and that all rats responded quicker on errors trials as they moved through subsequent subtasks within the session.

In contrast, the repeated measures ANOVA of latency to dig during postoperative error trials $($ Fig. 4D) revealed a significant interaction of Stage $\times$ Lesion_type, $F(6,132)=$ 26.81, $p<0.001$, a significant main effect of Stage, $F(6,132)=22.45, p<0.001$, and a significant main effect of Lesion-type, $F(1,22)=57.15, p<0.001$. To investigate the interaction effect, posthoc comparisons of the simple main effects (corrected for multiple comparisons) were computed. Postoperatively, MD lesion rats responded slower on error trials during the SD subtask, $t(22)=10.09, p<0.001$, the CD subtask, $t(22)=7.65, p<0.001$, and the REV1 subtask, $t(22)=4.44, p<0.001$. However, for the final reversal subtask, REV3, completed after the ED shift, MD lesion rats were quicker on the error trials than MD Sham controls, $t(22)=3.53, p=0.002$. This result in combination with the reduced trials to criterion required to complete this subtask (see Fig. 2A) suggests that the MD lesion rats may have experienced overtraining due to the number of additional trials to criterion required to adapt their choice responding in the ED subtask (Dhawan et al., 2019). For correct trials, repeated measures ANOVAs for the preoperative or postoperative session showed no significant main effects and no significant interaction ( $p$ 's $>0.05)$. 


\section{$414 \quad 4.0 \quad$ Discussion}

415 This set of experiments investigated the active influence of rat mediodorsal thalamus (MD) in

416 cognitive flexibility. More specifically, we examined whether rats with excitotoxic lesions of

417 the central and medial MD were impaired in performing an attentional set-shifting task that

418 measures intra-dimensional (ID) and extra-dimensional (ED) shifts in attention to sensory

419 discriminations (digging media or odors). Our new results indicate that rats with MD lesions

420 required more trials to criterion to learn the optimal response strategy during the initial

421 simple discrimination (SD). Although, once this stable response strategy had been learnt, MD

422 lesion rats were able to attain an attentional set strategy as they acquired further two-choice

423 discriminations involving the same sensory dimension during the CD and ID shifts, and

424 during reversals (REV1 and REV2) of the reward contingencies associated to the exemplars.

425 However, when the relevant sensory dimension was switched, for the ED subtask, the MD

426 lesion rats were markedly impaired and required many more trials to update their choices

427 using a new response strategy, as measured by the significant ED shift cost. Further, for

428 REV3, the third reversal performed after the ED shift, the MD lesion rats showed facilitated

429 learning, suggesting they had received 'overtraining' as a consequence of completing many

430 more trials to reach criterion in the ED subtask (see below). The analyses of behavioral

431 responses indicated that the MD lesion rats made significantly fewer correct $2^{\text {nd }}$ choice

432 within-trial responses suggesting that information transfer via the MD is critical for the rapid,

433 within-trial monitoring and updating of an optimal choice response. Interestingly, similar

434 updating deficits in choice responding have been previously reported in rhesus macaques

435 with magnocellular MD excitotoxic lesions (Chakraborty et al., 2016). 


\section{Learning and updating}

These behavioral data accord somewhat with previous studies conducted in different species. For example, marmosets with lateral PFC lesions (Dias et al., 1996) or rats with medial PFC lesions (equivalent frontal regions; Birrell and Brown, 2000) are also impaired at the ED subtask, showing a similar ED shift cost as our MD lesion rats. However, unlike our MD lesion rats, these marmosets and rats did not need more trials to reach criterion during the SD subtask. Instead, the MD lesion rats' learning deficit during the SD subtask accords with rats that had anterior thalamic nuclei (ATN) lesions, who similarly required more trials to acquire the SD subtask (Wright et al., 2015), although these ATN lesion rats were not impaired during the ED subtask. Instead, and in contrast to our MD lesion rats, rats with ATN lesions continued to be impaired during the CD subtask and subsequent ID shifts (Wright et al., 2015) indicating that they never properly learnt the discrimination rule, which suggests an intact ATN (a brain structure adjacent to the MD but with markedly different corticothalamo-cortical connectivity) is important for supporting the formation of an attentional set strategy.

As already indicated, with more trials, our rats with MD lesions were able to learn the SD subtask to criterion and apply the optimal rule during subsequent subtasks. For the SD subtask, trials revert back to presenting only one sensory dimension and two stimulus exemplars. Other studies also confirm MD lesion rats are impaired in acquisition of an initial task rule (e.g. Hunt and Aggleton, 1998). Other rodent studies causing temporary perturbations to the MD also show impaired discrimination learning (Ferguson and Gao, 2018; Courtiol et al., 2019). Thus these results from across species reinforce the notion that the MD is not simply mimicking the PFC (DeNicola et al., 2020; Miller et al., 2017; Mitchell et al., 2007; Mitchell and Gaffan 2008; Mitchell et al., 2014). Consequently the influence of other cortico-cortical interactions that also indirectly transfer information to the cortex via the 
MD and other thalamic structures, e.g. those PFC interactions with limbic structures in the temporal lobes and sensory association areas are also critical during learning sensory

463 discriminations (Banjeree et al., 2020; Bueno-Junior et al., 2018; Chakraborty et al., 2019;

464 Floresco and Grace, 2003; Mitchell, 2015; Pelekanos et al., 2020).

However, during the ED subtask, our MD lesion rats were markedly impaired at rapidly adapting this now well-established choice response strategy when they had to attend to the previously irrelevant (non-rewarded) sensory dimension. The demands on cognitive flexibility, response inhibition, and adapting behavioral responses after negative feedback are increased when the animals must make an ED shift (Dhwana et al., 2019). The increased effort involved in adapting to these changes in attention is measured by a shift cost, and it is expected that more trials to criterion are required during learning the ED than during learning the ID shift. While the ID/ED shift cost was higher for both groups (Fig. 2B), it was significantly increased for the MD lesion rats compared to the MD Sham controls. As

474 indicated, increased shift costs have also been observed in marmosets and rats with lesions to 475 the comparative PFC regions (Dias et al., 1996; Birrell and Brown, 2000). Surprisingly 476 though, rats with damage to the nucleus reuniens (Re), another thalamic structure located near the MD, which interconnects the medial PFC and hippocampus (Hoover and Vertes, 478 2007; Hoover and Vertes, 2011; Vertes et al., 2015) do not cause deficits in the ED subtask 479 or produce a significant ED shift cost. Instead, rats with NRe damage are impaired at 480 acquiring the attentional set strategy, similar to rats with ATN lesions (Linley et al., 2016). 481 Thus, our MD thalamus results are unique. Evidence from studies in mice suggests the MD supports the frontal cortex to sustain intra-cortical attentional control without transferring categorical information about a 484 particular task rule (Schmitt et al., 2017), or that medial PFC-MD projections are important 485 for behavioral flexibility but not task engagement (Marton et al., 2018; Nakayama et al., 
2018). Further, the MD has been shown to have a critical role in maintaining the balance between excitation and inhibition in dorsomedial PFC via its influence on interneurons

488 (Anastasiades et al., 2021; Ferguson and Gao, 2018) and pyramidal neurons (Collins et al.,

489 2018; Delevich et al., 2015). Our results are supportive of these circuit level interactions,

490 although our deficits indicate the influence of MD on frontal circuits is specific to certain

491 aspects of the attentional set-shifting task, e.g. linked to the ED shift and learning the optimal choice response strategy, but not to the ID shift or completing reversals. However, our study

493 involved permanent MD lesions, which are likely to have caused some adaptations across the

494 network, while the studies in mice likely involve short-term changes as temporary

495 inactivation of the MD was employed. Nevertheless, it may be proposed that after MD

496 perturbations, while the animal is still able to detect a change in the task demands (i.e. they are responsive to negative feedback), they are impaired at rapidly adapting their behavior and coordinating correct choice responses within a trial after a change to the already established choice response strategy (Block et al., 2007; Hunt and Aggleton, 1998; Chakraborty et al., 2016). Interestingly though, in both these rodent studies (Block et al., 2007; Hunt and Aggleton, 1998), the learning deficits were attributed to increased perseverative responding.

Others have also observed increased perseverative responding after MD perturbations

503 (Ferguson and Gao, 2018). In contrast, our current rodent work and previous work in rhesus

504 macaques has shown an intact MDmc is necessary for rapid reward guided learning of complex discriminations (Mitchell et al., 2007a; Chakraborty et al., 2016; Chakraborty et al., 2019), although the deficits linked to learning in monkeys are attributed to increased response switching (Mitchell et al., 2007a; Chakraborty et al., 2016). These congruent results across species after MD damage suggest that cortical information transfer via the MD is particularly important when rapid, within a trial changes in choice response strategies linked to establishing a new rule are required. 


\section{Reversal learning}

For the reversal subtasks (REV1 and REV2), somewhat surprisingly, our MD lesion rats were unimpaired during reversals in the reward contingencies. Rats and marmosets with comparable medial PFC damage are also not impaired in reveral learning subtasks (Dias et al., 1996; Birrell and Brown, 2000). Instead, OFC perturbations cause deficits in the reversal subtasks, while leaving performance intact during the SD and ED subtasks of the ID/ED task (Dias et al., 1997; McAlonan and Brown, 2003; Chase et al., 2012). These observations accord with a large body of research indicating that separate mammalian PFC subregions differentially contribute to learning, memory, and other cognitive functions (e.g. Clark et al, 2004; Rudebeck et al., 2008; for review, see Dalley, Cardinal and Robbins, 2004; Uylings, Groenewegen and Kolb, 2003; Izquierdo et al., 2017).

MD is reciprocally connected to the OFC and medial PFC (Groenwegen, 1988; Preuss, 1995; Ray and Price, 1992). Thus this double dissociation in deficits between these reciprocally interconnected $\mathrm{MD}-\mathrm{OFC}$ and $\mathrm{MD}$-medial $\mathrm{PFC}$ brain regions needs to be reconciled. First, it must be noted that the MD rats had experienced all of the subtasks (and thus were familiar with the concept of reversal learning and that it can appear in the task structure) during their preoperative test session, which may have reduced or eliminated the reversal learning deficit (Costa et al., 2015; Jang et al., 2015). Further, for the MD, while previous evidence of reversal learning deficits are reported for some tasks after MD perturbations (Block et al., 2007; Chudasama et al., 2001; Ferguson and Gao, 2018; Hunt and Aggleton, 1998; Ostlund and Balliene, 2008; Parnaudeau et al., 2013, 2015), the evidence is mixed (Beracochea et al., 1989; Alcaraz et al., 2018; Fresno et al., 2019). These mixed effects may be the consequence of differences in task structure and the way that the reversal is introduced. Other factors, including the extent of disruption caused to thalamic structures adjacent to the MD may also be a factor (Mitchell and Chakraborty, 2013; Wolff and Vann, 
2019). Indeed, one recent study has identified that another thalamic structure nearby to the MD - the submedius thalamus - may contribute a role in reversal learning, instead of the MD

538 (Fresno et al., 2019). Consequently, our current results and this above evidence highlights

539 that the information transfer between OFC and MD is not always critical for reversal learning

540 per se. Taken together, it is clear that the MD is not simply mimicking the behavioral deficits observed after PFC damage, and instead shows that the MD and different subregions of PFC are supporting different aspects of cognitive flexibility. At least in monkeys, OFC-MDmc interactions are critical for adaptive, value based decision-making (Browning et al., 2015;

544 Izquierdo and Murray, 2010; Mitchell et al., 2007b), while in rodent studies, the OFC-striatal part of the cortico-striatal-thalamic neural circuits have been implicating in reversal learning (Gremel and Costa, 2013; Izqueirdo et al., 2017; Nakayama et al 2018; Yang et al., 2020; Yin et al., 2005) or OFC-submedius thalamus circuits (Fresno et al., 2019). Other OFC-striatalthalamic and OFC-thalamic interactions are potentially more involved in supporting reversal learning. In addition, thalamic inputs from the intralaminar thalamic nuclei and motor thalamus to the striatum contribute a selective role in inhibitory control and behavioral flexibility (e.g. Saund et al., 2017).

Intriguingly, our additional analyses used to investigate the types of behavioral deficits occurring after MD lesions showed that our rats made fewer correct $2^{\text {nd }}$ choice within-trial responses. Given that our MD lesion rats required more trials to criterion to learn the SD and ED subtasks, this change in behavioral responses suggests that without the MD thalamus, our rats could not rapidly adapt their choice responses as the trial was progressing (within-trial) after the changed task demands. However, this reduced correct $2^{\text {nd }}$ choice within-trial responding does not suggest the rats adopted perseverative responding (they would have potentially been impaired in reversal learning if they had) or were not learning about the stimulus and associated rewards. We can conclude this because, after acquiring the 
ED shift, the MD lesion rats required fewer trials to criterion to learn the final reversal

562

563

564

565

566

567

568

569

570

571

572

573

574

575

576

577

578

579

580 subtask, REV3. This facilitation of learning during the final reversal subtask suggests that the MD lesion rats had experienced 'overtraining' on all of the stimulus features associated with each sensory dimension as they required more trials to learn the ED subtask (Dhawan et al., 2019). It is well established that overtraining rats during discrimination learning can eliminate any reversal learning deficits (Reid, 1953; Lovejoy, 1966; Nilsson et al., 2015 for review). As Pearce and Mackintosh (2010) indicate, until learning is fully consolidated, all stimulus features continue to be attended to and this seemed to be so for our rats, even with the MD lesion. Thus, we propose, in accord with others, that through experiencing these extra trials during the ED subtask, the MD lesion group may have developed a greater understanding of the rewarded and unrewarded stimulus dimensions, thus increasing the salience of the predictive cues and reducing/eliminating the number of factors that can led to an error. In essence, these extra trials in the ED subtask helped our MD lesion rats to slowly learn the optimal response strategy so when the final reversal was encountered, they were readily able to continue to implement this optimal response strategy for the sensory dimension while simply reversing the two stimulus exemplars (something they were already well-practiced at doing). An MD lesion does not render an animal or human densely amnesic or cause them to be insensitive to the receipt of rewards (Mitchell, 2015). Instead, we are proposing that after the loss of the $\mathrm{MD}$, an animal or human is unable to rapidly learn a new choice response strategy or rapidly update a well-established optimal one.

However, in the Birrell and Brown (2000) study, rats with medial PFC lesions did not show a facilitation of learning during the final reversal subtask after showing a similar ED shift cost to our MD lesion rats. We cannot be certain why medial PFC lesion rats also did not show a facilitation of learning in the final reversal subtask, although exploring whether they experience overtraining is worthy of further investigation. What is known is that the 
medial PFC is proposed to contribute to learning via supporting the processing of error feedback related to adapting stimulus-action selection. For example, during instrumental learning, medial PFC lesions in rats contribute to the acquisition but not the expression of goal-directed actions (Ostlund and Balleine, 2005).

lesion rats. With the ID/ ED task, it is important to establish that the animals understand the relevant sensory dimension that is rewarded across the related subtasks within the session.

593 Fortunately, in our animals, this transfer of knowledge was evident as the MD lesion rats

594 showed reliable learning in the subtasks, CD, REV1, ID and REV2, that required the implementation of choice responses (follow the same rule) to the same sensory dimension as the SD to receive reward. Further, both Sham controls and MD lesion rats showed similar numbers of trials to criterion during the REV1 and REV2 subtask, which indicates that they

598 did not favor one feature of the stimulus dimension more than the other or that they were 599 insensitive to negative feedback. However, we only used male rats in this current study so the results might not transfer to female rats. Additionally, the MD is a subcortical node in the olfactory neural circuitry that also includes the OFC (Courtiol et al., 2015; Veldhuizen et al., 2020). However, evidence collected from rodents with MD perturbations or humans with strokes affecting the MD indicates that changes to the MD do not impair olfactory

604 discriminations (Courtiol et al., 2019; Tham et al., 2011). Moreover, our MD lesion rats 605 showed similar levels of olfactory discriminations as the MD Sham controls (see Material and Methods for further details).

Finally, the diffuse influence of the MD thalamocortical inputs to several frontal cortex structures (Alcaraz et al., 2016; Kuramoto et al., 2017; Mitchell, 2015; Wolff and

609 Vann, 2019) supports previous findings implicating medial PFC-MD connectivity in flexible 610 behaviors (Marton et al., 2018; Nakayama et al., 2018). Furthermore, the MD along with the 
611 OFC provides additional evidence for the contribution of these areas in supporting online,

612 'here and now' reward-guided learning and decision-making (Gardner et al., 2017; Gardner

613 et al., 2019; Schoenbaum et al., 2011; Wallis, 2007). Intact OFC connectivity is essential to

614 the animals' ability to perform reward-guided learning in order that other interconnected

615 neural networks, likely including cortico-cortical, cortico-striatal, and cortico-thalamo-

616 cortical connectivity, can determine the optimal choice responses and implement the

617 appropriate actions (Browning et al 2015; Chakraborty et al 2016; Nakayama et al 2018;

618 Perry et al., 2021; Rushworth et al., 2011).

619 The ID/ ED task is analogous to the Wisconsin Card Sorting Test (WCST) in humans.

620 In healthy humans performing the WCST during neuroimaging, the MD is activated during

621 responding to negative feedback after the choice has been executed (Monchi et al., 2001).

622 Unfortunately, thus far, humans with stroke damage in the MD have cognitive deficits that

623 are clinically very poorly defined (Pergola et al., 2018). However, people diagnosed with

624 Alzheimer's disease (AD), Parkinson's disease (PD), or schizophrenia show impaired

625 attentional set-shifting performance (e.g. Barch et al., 2009; Monchi et al., 2004; Owen et al,

626 1993). Neuroimaging and post-mortem studies show marked changes in the MD and/ or ATN

627 in these diseases (Hornberger et al., 2012; Ouhaz et al., 2018; Pergola et al., 2015; Perry et

628 al., 2018). Our current evidence advocates for studies investigating cortico-thalamocortical

629 transfer of information in people diagnosed with AD combined with more frontal pathology,

630 or PD, or schizophrenia.

631 To conclude, excitotoxic damage to the rodent central and medial MD selectively

632 increased the trials to criterion on the ED subtask, producing a shift cost. This selective

633 performance deficit is similar to monkeys with lateral PFC inactivation (Dias et al., 1996;

634 Dias et al., 1997) and rats with medial PFC lesions (Birrell and Brown, 2000). Further, this

635 deficit after MD lesions contrasts with monkeys or rats with perturbations to the OFC, who 
636 are selectively impaired on reversal learning, but not on ED shifts (Chase et al., 2012; Dias et 637 al., 1997; McAlonan and Brown, 2003). As evidenced, the frontal cortex is critically involved 638 in value-based decision-making and reward-guided learning (Miller, 2000; Rushworth et al. 639 2011; Wallis, 2007). However, cortico-thalamocortical connections also contribute a role 640 (Browning et al. 2015; Chakraborty et al., 2016; Izqueirdo and Murray, 2010; Mitchell et al., 641 2014; Pelekanos et al., 2020). In rodents, the MD and the medial PFC together appear crucial 642 for binding reward information and behavior (Bradfield et al., 2013; Corbit et al., 2003), as 643 inhibition of dorsomedial PFC-projecting MD neurons results in rats having difficulties with 644 tracking changes in action-outcome contingencies (Alcaraz et al., 2018). In the current study, 645 we show that rat medial and central MD are critical for rapidly updating an optimal choice 646 response strategy. That is, when the MD is damaged, there is a diminished ability to rapidly 647 learn (within a trial) a choice response strategy, as well as update a well-established one as 648 task demands change. Behaviorally, our MD lesion rats made less correct $2^{\text {nd }}$ choice within649 trial responses, suggesting they could not rapidly alter their choice response strategy when 650 they encountered the unrewarded stimuli on a given trial. 
651

652

653

654

655

656

657

658

659

660

661

662

663

664

665

666

667

668

669

670

671

672

673

674

\section{References}

Alcaraz F, Fresno V, Marchand AR, Kremer EJ, Coutureau E, Wolff M (2018)

Thalamocortical and corticothalamic pathways differentially contribute to goal-directed behaviors in the rat. eLife, 7, e32517. 10.7554/eLife.32517

Alcaraz F, Marchand AR, Courtand G, Coutureau E, Wolff M (2016) Parallel inputs from the mediodorsal thalamus to the prefrontal cortex in the rat. Eur J Neurosci. 44(3):19721986. 10.1111/ejn.13316.

Andrews J, Wang L, Csernansky JG, Gado MH, Barch DM (2006) Abnormalities of thalamic activation and cognition in schizophrenia. Am J Psychiatry. 163(3):463-469. 10.1176/appi.ajp.163.3.463.

Banerjee A, Parente G, Teutsch J, Lewis C, Voigt FF, Helmchen F (2020) Value-guided remapping of sensory cortex by lateral orbitofrontal cortex. Nature 585, 245-250. https://doi.org/10.1038/s41586-020-2704-z

Barch DM, Braver TS, Carter CS, Poldrack RA, Robbins TW (2009) CNTRICS Final Task Selection: Executive Control, Schizophrenia Bulletin, 35: 115-135, 10.1093/schbul/sbn154

Beracochea DJ, Jaffard R, Jarrard LE (1989) Effects of anterior or dorsomedial thalamic ibotenic lesions on learning and memory in rats. Behav Neural Biol. 51(3):364-376. 10.1016/s01631047(89)91000-5.

Birrell JM, Brown VJ (2000) Medial frontal cortex mediates perceptual attentional set shifting in the rat. $J$ Neurosci. 20(11):4320-4324.

Bissonette GB, Martins GJ, Franz TM, Harper ES, Schoenbaum G, Powell EM (2008) Double dissociation of the effects of medial and orbital prefrontal cortical lesions on attentional and affective shifts in mice. $J$ Neurosci. 28:11124-11130. 10.1523/JNEUROSCI.282008.2008. 
Block AE, Dhanji H, Thompson-Tardif SF, Floresco SB (2007) Thalamic-Prefrontal CorticalVentral Striatal Circuitry Mediates Dissociable Components of Strategy Set Shifting, Cerebral Cortex. 17(7): 1625-1636, 10.1093/cercor/bhl073

Bradfield LA, Hart G, Balleine BW (2013) The role of the anterior, mediodorsal, and parafascicular thalamus in instrumental conditioning. Front Systems Neurosci. 7, 51. 10.3389/fnsys.2013.00051

Brown VJ, Tait DS (2016) Attentional Set-Shifting Across Species. Curr Top Behav Neurosci.28:363-395.10.1007/7854_2015_5002.

Browning PGF, Chakraborty S, Mitchell AS (2015) Evidence for mediodorsal thalamus and prefrontal cortex interactions during cognition in macaques. Cereb Cortex. 25, 45194534.

Bueno-Junior LS, Leite JP (2018) Input Convergence, Synaptic Plasticity and Functional Coupling Across Hippocampal-Prefrontal-Thalamic Circuits. Front Neural Circuits, 12, 40. 10.3389/fncir.2018.00040

Chakraborty S, Kolling N, Walton ME, Mitchell AS (2016) Critical role for the mediodorsal thalamus in permitting rapid reward-guided updating in stochastic reward environment. eLife, 5:e13588. 10.7554/eLife.13588

Chakraborty S, Ouhaz Z, Mason S, Mitchell AS (2019) Macaque parvocellular mediodorsal thalamus: dissociable contributions to learning and adaptive decision-making. Eur $J$ Neurosci. 49: 1041-1054. doi: 10.1111/ejn.14078.

Chase EA, Tait DS, Brown VJ (2012) Lesions of the orbital prefrontal cortex impair the formation of attentional set in rats. Eur J Neurosci. 36:2368-2375. 10.1111/j.1460-9568.2012.08141.x. 
Chudasama Y, Bussey TJ, Muir JL (2001) Effects of selective thalamic and prelimbic cortex lesions on two types of visual discrimination and reversal learning. Eur J Neurosci. 14: 1009-1020. 10.1046/j.0953-816x.2001.01607.x.

Clark L, Cools R, Robbins TW (2004) The neuropsychology of ventral prefrontal cortex: decision-making and reversal learning. Brain Cogn. 55: 41-53. doi: 10.1016/S02782626(03)00284-7.

Costa VD, Tran VL, Turchi J, Averbeck BB (2015) Reversal Learning and Dopamine: A Bayesian Perspective. J. Neurosci. 35: 2407-2416; DOI:10.1523/JNEUROSCI.198914.2015

Collins DP, Anastasiades PG, Marlin JJ, Carter AG (2018) Reciprocal circuits linking the prefrontal cortex with dorsal and ventral thalamic nuclei. Neuron, 98: 366-379.e4. 10.1016/j.neuron.2018.03.024

Corbit LH, Muir JL, Balleine BW (2003) Lesions of mediodorsal thalamus and anterior thalamic nuclei produce dissociable effects on instrumental conditioning in rats. Eur. J. Neurosci. 18, 1286-1294. 10.1046/j.1460-9568.2003.02833.x

Courtiol E, Neiman M, Fleming G, Teixeira CM, Wilson DA (2019) A specific olfactory cortico-thalamic pathway contributing to sampling performance during odor reversal learning. Brain Struct Funct. 224, 961-971. 10.1007/s00429-018-1807-x

Courtiol E, Wilson DA (2015) The olfactory thalamus: unanswered questions about the role of the mediodorsal thalamic nucleus in olfaction. Front Neural Circuits. 9, 49. 10.3389/fncir.2015.00049

Dajani DR, Uddin LQ (2015) Demystifying cognitive flexibility: Implications for clinical and developmental neuroscience. Trends Neurosci. 8: 571-578. doi:

10.1016/j.tins.2015.07.003 
Dalley JW, Cardinal RN, Robbins TW (2004) Prefrontal executive and cognitive functions in rodents: neural and neurochemical substrates. Neurosci Biobehav Rev. 28: 771-84. doi: 10.1016/j.neubiorev.2004.09.006.

DeNicola AL, Park MY, Crowe DA, MacDonald AW 3rd, Chafee MV (2020) Differential Roles of Mediodorsal Nucleus of the Thalamus and Prefrontal Cortex in DecisionMaking and State Representation in a Cognitive Control Task Measuring Deficits in Schizophrenia. J Neurosci. 40, 1650-1667. 10.1523/JNEUROSCI.1703-19.2020

Dhawan SS, Tait DA, Brown VJ (2019) More rapid reversal learning following overtraining in the rat is evidence that behavioural and cognitive flexibility are dissociable. Behavioural Brain Research, 363: 45-52. 10.1016/j.bbr.2019.01.055

Dias R, Robbins TW, Roberts AC (1996) Dissociation in prefrontal cortex of affective and attentional shifts. Nature 380: 69-72. 10.1038/380069a0

Dias R, Robbins TW, Roberts AC (1997) Dissociable forms of inhibitory control within prefrontal cortex with an analog of the Wisconsin Card Sort Test: restriction to novel situations and independence from "on-line" processing. J Neurosci 17: 9285-97

Ferguson BR, Gao WJ (2018) PV Interneurons: Critical Regulators of E/I Balance for Prefrontal Cortex-Dependent Behavior and Psychiatric Disorders. Front Neural Circuits. 12:37. 10.3389/fncir.2018.00037

Floresco SB, Grace AA (2003) Gating of hippocampal-evoked activity in prefrontal cortical neurons by inputs from the mediodorsal thalamus and ventral tegmental area. $J$ Neurosci. 23: 3930-3943. 10.1523/JNEUROSCI.23-09-03930.2003

Fresno V, Parkes SL, Faugère A, Coutureau E, Wolff M (2019) A thalamocortical circuit for updating action-outcome associations. Elife. 8:e46187. doi: 10.7554/eLife.46187 
Gardner MPH, Conroy JC, Sanchez DC, Zhou J, Schoenbaum G (2019) Real-Time Value Integration during Economic Choice Is Regulated by Orbitofrontal Cortex. Curr Biol. 29: 4315-4322 .e4. 10.1016/j.cub.2019.10.058

Gardner MPH, Conroy JS, Shaham MH, Styer CV, Schoenbaum G (2017) Lateral Orbitofrontal Inactivation Dissociates Devaluation-Sensitive Behavior and Economic Choice. Neuron. 96: 1192-1203.e4. 10.1016/j.neuron.2017.10.026

Giraldo-Chica M, Rogers BP, Damon SM, Landman BA, Woodward ND (2018) Prefrontalthalamic anatomical connectivity and executive cognitive function in schizophrenia. Biol Psychiatry. 83: 509-517. 10.1016/j.biopsych.2017.09.022

Gremel C, Costa R (2013) Orbitofrontal and striatal circuits dynamically encode the shift between goal-directed and habitual actions. Nat Commun 4: 2264 https://doi.org/10.1038/ncomms3264

Groenewegen HJ (1988) Organisation of the afferent connections of the mediodorsal thalamic nucleus in the rat, related to the mediodorsal prefrontal topography. Neuroscience 24 , $379-431.10 .1016 / 0306-4522(88) 90339-9$

Guillery RW, Sherman SM (2011) Branched thalamic afferents: what are the messages that they relay to the cortex? Brain Res Rev. 66: 205-219. 10.1016/j.brainresrev.2010.08.001

Halassa MM, Kastner S (2017) Thalamic functions in distributed cognitive control. Nat Neurosci. 20: 1669-1679. doi: 10.1038/s41593-017-0020-1.

Hoover WB, Vertes RP (2007) Anatomical analysis of afferent projections to the medial prefrontal cortex in the rat. Brain Struct Funct. 212: 149-79. 10.1007/s00429-0070150-4.

Hoover WB, Vertes RP (2011) Projections of the medial orbital and ventral orbital cortex in the rat. J Comp Neurol. 519:3766-3801. 10.1002/cne.22733 pmid:21800317 
Hornberger M, Wong S, Tan R, Irish M, Piguet O, Kril J, Hodges JR, Halliday G (2012) In

770 vivo and post-mortem memory circuit integrity in frontotemporal dementia and Alzheimer's disease. Brain. 135: 3015-3025. 10.1093/brain/aws239

Hunt PR, Aggleton JP (1998) Neurotoxic lesions of the dorsomedial thalamus impair the acquisition but not the performance of delayed matching to place by rats: a deficit in shifting response rules. J Neurosci, 18: 10045-10052.

Izqueirdo A, Brigman JL, Radke AK, Rudebeck PH, Holmes A (2017) The neural basis of reversal learning: An updated perspective. Neuroscience. 345, 12-26. 10.1016/j.neuroscience.2016.03.021

Izquierdo A, Murray EA (2010) Functional interaction of medial mediodorsal thalamic nucleus but not nucleus accumbens with amygdala and orbital prefrontal cortex is essential for adaptive response selection after reinforcer devaluation. J Neurosci. 30: 661-669. doi:10.1523/JNEUROSCI.3795-09.2010.

Jang AI, Costa VD, Rudebeck PH, Chudasama Y, Murray EA, Averbeck BB (2015) The Role of Frontal Cortical and Medial-Temporal Lobe Brain Areas in Learning a Bayesian Prior Belief on Reversals. J Neurosci. 35: 11751-11760. DOI:10.1523/JNEUROSCI.1594-15.2015

Krettek JE, Price JL (1977) Cortical projections of the mediodorsal nucleus and adjacent thalamic nuclei in the rat. J Comp Neurol. 171: 157-191. doi:10.1002/cne.901710204

Koechlin E, Ody C, Kouneiher F (2003) The architecture of cognitive control in the human prefrontal cortex. Science, 302: 1181-1185.

Linley, SB, Gallo, MM, Vertes, RP (2016) Lesions of the ventral midline thalamus produce deficits in reversal learning and attention on an odor texture set shifting task. Brain Res, 1649: 110-122. doi:10.1016/j.brainres.2016.08.022

Lindgren HS, Wickens R, Tait DS, Brown VJ, Dunnett SB (2013) Lesions of the 
dorsomedial striatum impair formation of attentional set in rats. Neuropharmacology. 71: 148-153. doi:10.1016/j.neuropharm.2013.03.034.

Lovejoy E (1966) Analysis of the overlearning reversal effect. Psychological Review, 73: 87103, doi:10.1037/h0022687.

Marton TF, Seifikar H, Luongo FJ, Lee AT, Sohal VS (2018) Roles of Prefrontal Cortex and Mediodorsal Thalamus in Task Engagement and Behavioral Flexibility. J Neurosci. 38: 2569-2578. 10.1523/JNEUROSCI.1728-17.2018.

McAlonan K, Brown VJ (2003) Orbital prefrontal cortex mediates reversal learning and not attentional set shifting in the rat. Behav Brain Res. 146: 97-103.

$$
\text { 10.1016/j.bbr.2003.09.019 pmid:14643463 }
$$

McGaughy J, Ross RS, Eichenbaum H (2008) Noradrenergic, but not cholinergic, deafferentation of prefrontal cortex impairs attentional set- shifting. Neuroscience. 153: 63-71. 10.1016/j.neuroscience.2008.01.064

Miller EK (2000) The prefrontal cortex and cognitive control. Nat. Rev. Neurosci. 1, 59-65

Miller RLA, Francoeur MJ, Gibson BM, Mair RG (2017) Mediodorsal Thalamic Neurons Mirror the Activity of Medial Prefrontal Neurons Responding to Movement and Reinforcement during a Dynamic DNMTP Task. eNeuro. 4(5):ENEURO.0196-17.2017. 10.1523/ENEURO.0196-17.2017.

Mitchell AS (2015) The mediodorsal thalamus as a higher order thalamic relay nucleus important for learning and decision-making. Neurosci BioBehav Rev 54: 76-88

Mitchell AS, Baxter MG, Gaffan D (2007a) Dissociable performance on scene memory learning and strategy implementation after lesions to magnocellular mediodorsal thalamic nucleus. J. Neurosci, 27: 11888-11895. 
Mitchell AS, Browning PGF, Baxter MG (2007b) Neurotoxic lesions of the medial mediodorsal nucleus of the thalamus disrupt reinforcer devaluation effects in rhesus monkeys. J. Neurosci, 27: 11289-11295.

Mitchell AS, Dalrymple-Alford JC (2005) Dissociable memory effects after medial thalamus lesions in the rat. Eur J Neurosci, 22: 973-985.

Mitchell AS, Gaffan D (2008) The primate magnocellular mediodorsal thalamus is necessary for memory acquisition, not for retrieval. J Neurosci, 28: 258-263.

Mitchell AS, Sherman SM, Sommer M, Mair R, Vertes R, Chudasama Y (2014) Advances in understanding mechanisms of thalamic relays in cognition and behavior. $J$ Neurosci 34 : $15340-15346$.

Monchi O, Petrides M, Petre V, Worsley K, Dagher A (2001) Wisconsin Card Sorting revisited: distinct neural circuits participating in different stages of the task identified by event-related functional magnetic resonance imaging. J Neurosci. 21: 7733-7741. 10.1523/JNEUROSCI.21-19-07733.2001.

Monchi O, Petrides M, Doyon J, Postuma RB, Worsley K, Dagher A (2004) Neural bases of set-shifting deficits in Parkinson's disease. J Neurosci. 24: 702-710. 10.1523/JNEUROSCI.4860-03.2004.

Nakayama H, Ibañez-Tallon I, Heintz N (2018) Cell-Type-Specific Contributions of Medial Prefrontal Neurons to Flexible Behaviors. J Neurosci. 38: 4490-4504. doi: 10.1523/JNEUROSCI.3537-17.2018.

Newman LA, McGaughy J (2011) Attentional effects of lesions to the anterior cingulate cortex: how prior reinforcement influences distractibility. Behav Neurosci. 125: 360371. doi:10.1037/a0023250 
Nilsson SRO, Alsiö J, Somerville EM, Clifton PG (2015) The rat's not for turning:

Dissociating the psychological components of cognitive inflexibility. Neurosci

Biobehav Rev, 56: 1-14, doi:10.1016/j.neubiorev.2015.06.015.

843 Ouhaz Z, Fleming H, Mitchell AS (2018) Cognitive functions and neurodevelopmental

844 disorders involving the prefrontal cortex and mediodorsal thalamus. Front Neurosci, 12,

33. $10.3389 /$ fnins. 2018.00033

Ostlund SB, Balleine BW (2005) Lesion of the medial prefrontal cortex disrupt the acquisition but not he expression of goal-directed learning. J. Neurosci. 25, 7763-7770.

Ostlund SB, Balleine BW (2008) Differential involvement of the basolateral amygdala and mediodorsal thalamus in instrumental action selection. J. Neurosci. 28, 4398-4405. 10.1523/jneurosci.5472-07.2008

Owen AM, Roberts AC, Hodges JR, Summers BA, Polkey CE, Robbins TW (1993) Contrasting mechanisms of impaired attentional set-shifting in patients with frontal lobe damage or Parkinson's disease. Brain. 116: 1159-1175. 10.1093/brain/116.5.1159.

Parnaudeau S, O'Neill PK, Bolkan SS, Ward RD, Abbas AI, Roth BL, Balsam PD, Gordon JA, Kellendonk C (2013) Inhibition of mediodorsal thalamus disrupts thalamofrontal connectivity and cognition. Neuron. 77: 1151-1162. 10.1016/j.neuron.2013.01.038.

Parnaudeau S, Taylor K, Bolkan SS, Ward RD, Balsam PD, Kellendonk C (2015) Mediodorsal thalamus hypofunction impairs flexible goal-directed behavior. Biol

Paxinos G, Watson C (2008) The rat brain in stereotaxic coordinates. Academic Press;

862 Pearce JM, Mackintosh NJ (2010) Two theories of attention: a review and a possible integration. In CJ Mitchell, ML Le Pelley (Eds). Attention and Associative Learning: From Brain to Behaviour. Oxford University Press. 
Pelekanos V, Premereur E, Mitchell DJ, Chakraborty S, Mason S, Lee ACH, Mitchell AS (2020) Cortico-cortical and thalamocortical changes in functional connectivity and white matter structural integrity after reward guided learning of visuospatial discriminations in rhesus monkeys. J Neurosci. 40: 7887-7901. 10.1523/JNEUROSCI.0364-20.2020

Pergola G, Danet L, Pitel AL, Carlesimo GA, Segobin S, Pariente J, Suchan B, Mitchell AS, Barbeau EJ (2018) The human mediodorsal thalamus: a regulator of prefrontal activity patterns and cognition. Trends Cogn Sci. pii: S1364-6613(18)30205-5. 10.1016/j.tics.2018.08.006.

Pergola G, Selvaggi P, Trizio S, Bertolino A, Blasi G (2015) The role of the thalamus in schizophrenia from a neuroimaging perspective. Neurosci Biobehav Rev. 54: 57-75. doi:10.1016/j.neubiorev.2015.01.013

Perry BAL, Lomi E, Mitchell AS (2021) Thalamocortical interactions in cognition and disease: The mediodorsal and anterior thalamic nuclei. Neurosci Biobehav Rev. 130: 162-177. doi: 10.1016/j.neubiorev.2021.05.032.

Perry JC, Pakkenberg B, Vann SD (2019) Striking reduction in neurons and glial cells in anterior thalamic nuclei of older patients with Down syndrome. Neurobiology of aging, 75: 54-61.

Preuss TM (1995) Do Rats Have Prefrontal Cortex? The Rose-Woolsey-Akert Program Reconsidered. J Cog Neurosci. 7: 1-24

Ray JP, Price JL (1992) The organization of the thalamocortical connections of the mediodorsal thalamic nucleus in the rat, related to the ventral forebrain-prefrontal cortex topography. J Comp Neurol. 323: 167-197. 
Regeur L, Pakkenberg B (1989) Optimizing sampling designs for volume measurements of components of human brain using a stereological method. J Microsc. 155: 113-121. doi: 10.1111/j.1365-2818.1989.tb04300.x.

Reid LS (1953) The development of noncontinuity behavior through continuity learning. $J$ Exp. Psyc. 46: 107-112. https://doi.org/10.1037/h0062488

Rougier NP, Noelle DC, Braver TS, Cohen JD, O'Reilly RC (2005) Prefrontal cortex and flexible cognitive control: Rules without symbols. Proc Natl Acad Sci USA, 102: 73387343.

Rudebeck PH, Behrens TE, Kennerley SW, Baxter MG, Buckley MJ, Walton ME, Rushworth MF (2008) Frontal cortex subregions play distinct roles in choices between actions and stimuli. J Neurosci, 28: 13775-13785. doi: 10.1523/JNEUROSCI.354108.2008 .

Rushworth MFS, Noonan MP, Boorman ED, Walton ME, Behrens TE (2011) Frontal cortex and reward-guided learning and decision-making. Neuron 70: 1054-1069.

Saund J, Dautan D, Rostron C, Urcelay GP, Gerdjikov TV (2017) Thalamic inputs to dorsomedial striatum are involved in inhibitory control: evidence from the five-choice serial reaction time task in rats. Psychopharmacology (Berl). 234: 2399-2407. doi:10.1007/s00213-017-4627-4

Schmitt L, Wimmer R, Nakajima M, Happ M, Mofakham S, Halassa MM (2017) Thalamic amplification of cortical connectivity sustains attentional control. Nature. 545: 219223. $10.1038 /$ nature22073

Schoenbaum G, Takahashi Y, Liu TL, McDannald MA (2011) Does the orbitofrontal cortex signal value?. Ann N Y Acad Sci. 1239: 87-99. doi:10.1111/j.1749-6632.2011.06210.x 
911 Sherman SM, Guillery RW (2002) The role of the thalamus in the flow of information to the cortex. Philos Trans R Soc Lond Ser B Biol Sci. 357: 1695-1708. $10.1098 /$ rstb.2002.1161

914 Tait DS, Chase EA, Brown VJ (2014) Attentional set-shifting in rodents: a review of 915 behavioural methods and pharmacological results. Curr Pharm Des. 20(31):5046-59.

916 Tait DS, Bowman EM, Neuwirth LS, Brown VJ (2018) Assessment of intradimensional/ 917 extradimensional attentional set-shifting in rats. Neurosci Biobehav Rev. 89: 72-84. doi: 10.1016/j.neubiorev.2018.02.013.

Tham WWP, Stevenson RJ, Miller LA (2011) The role of the mediodorsal thalamic nucleus in human olfaction. Neurocase 17: 148-159. 10.1080/13554794.2010.504728

Uylings HB, Groenewegen HJ, Kolb B (2003) Do rats have a prefrontal cortex? Behav Brain Res. 146: 3-17. doi: 10.1016/j.bbr.2003.09.028.

Vertes RP, Linley SB, Hoover WB (2015) Limbic circuitry of the midline thalamus. Neurosci Biobehav Rev. 54: 89-107. doi: 10.1016/j.neubiorev.2015.01.014.

Wallis JD (2007) Orbitofrontal cortex and its contribution to decision-making. Annu. Rev. Neurosci. 30: 31-56.

Wright NF, Vann SD, Aggleton JP, Nelson AJ (2015) A critical role for the anterior thalamus in directing attention to task-relevant stimuli. J Neurosci. 35: 5480-5488.

930 Yang Z, Wu G, Liu M, Sun X, Xu Q, Zhang C, Lei H (2020) Dysfunction of orbitofrontal 931 GABAergic interneurons leads to impaired reversal learning in a mouse model of obsessive-compulsive disorder. Curr Biol, 31: 381-393.e4. doi.org/10.1016/j.cub.2020.10.045. 
934 Yin HH, Ostlund SB, Knowlton BJ, Balleine BW (2005) The role of the dorsomedial

935 striatum in instrumental conditioning. Eur J Neurosci. 22: 513-523. 10.1111/j.1460-

$936 \quad 9568.2005 .04218 . x$ 
939 Figure 1. A. Photomicrographs of the thalamus in a MD Sham control rat (top) and a MD

940 lesion rat (bottom). B. A series of coronal schematics throughout the mediodorsal thalamus

941 showing the area of cell loss in the MD lesion group. Numbers refer to the distance from

942 bregma (Paxinos and Watson, 2008). Abbreviations: 3V: third ventricle; dLGN: dorsal lateral

943 geniculate nucleus; LHb: lateral habenula; LD: laterodorsal thalamus; MD: mediodorsal

944 thalamus; MHb: medial habenula; PV: paraventricular nucleus; VPM: ventral posterior

945 medial thalamus.

947 Figure 2. Mean (+SEM) trials to criterion data for the attentional set-shifting task. (A)

948 Preoperative test session. (B) Postoperative test session. MD lesion rats were significantly

949 slower to learn the SD and the ED subtasks compared to MD Sham controls $\left({ }^{*} p<0.001\right)$. MD

950 Sham control rats, like MD lesion rats, needed more trials to learn the ED, as expected.

951 However, the MD lesion rats required significantly fewer trials to criterion to learn REV3,

952 the reversal occurring immediately after the ED, compared to the MD Sham controls $\left({ }^{*} p\right.$

953 <0.001). Also see the Extended Data Figure 2-1 showing boxplots of the mean trials to

954 criterion for individual rats during the postoperative session of the attentional set-shifting

955 task.

956

957 Extended Data Figure 2-1 showing boxplots of the mean trials to criterion for individual

958 rats during the postoperative session of the attentional set-shifting task. MD lesion rats took

959 significantly more trials to learn the SD and the ED subtasks compared to MD Sham controls

$\left.960 \quad{ }^{*} p<0.001\right)$. MD Sham control rats also needed more trials to learn the ED, as expected. The

961 MD lesion rats required significantly fewer trials to learn REV3, the reversal occurring 
962 immediately after the ED, compared to the MD Sham controls $(* p<0.001)$. Box shows $1^{\text {st }}$

963 and $3^{\text {rd }}$ quartile and whiskers are the minimum and maximum trials to criterion for individual

964 rats.

965

966

967 Figure 3. (A) Mean (+SEM) trials to criterion shift cost data for the ED stage for

968 preoperative (Pre) and postoperative (Post) testing sessions. The shift cost is computed as the

969 number of trials to criterion in the ED minus the number of trials to criterion in the ID. Both

970 groups of rats showed a shift cost during the ED, when they had to learn to attend to the

971 previously irrelevant dimension. However, the shift cost for the MD lesion rats in the

972 postoperative test session was significantly different to the MD Sham controls. (B) Mean

973 (+SEM) percent correct $2^{\text {nd }}$ choice within trial responses made during the preoperative and

974 postoperative performance sessions of the attentional set-shifting task. MD lesion rats made

975 fewer correct $2^{\text {nd }}$ choice within trial responses than MD Sham controls $(* p$ 's $=0.001)$.

976

977 Figure 4. Mean (+SEM) latencies to dig (secs) for for MD Sham controls and MD lesion rats

978 during each subtask of the ID/ED attentional set shifting task (A) Preoperative correct trials.

979 (B) Postoperative correct trials. (C) Preoperative error trials. (D) Postoperative error trials (**

$980 \quad p<0.001 ; * p=0.002)$. 


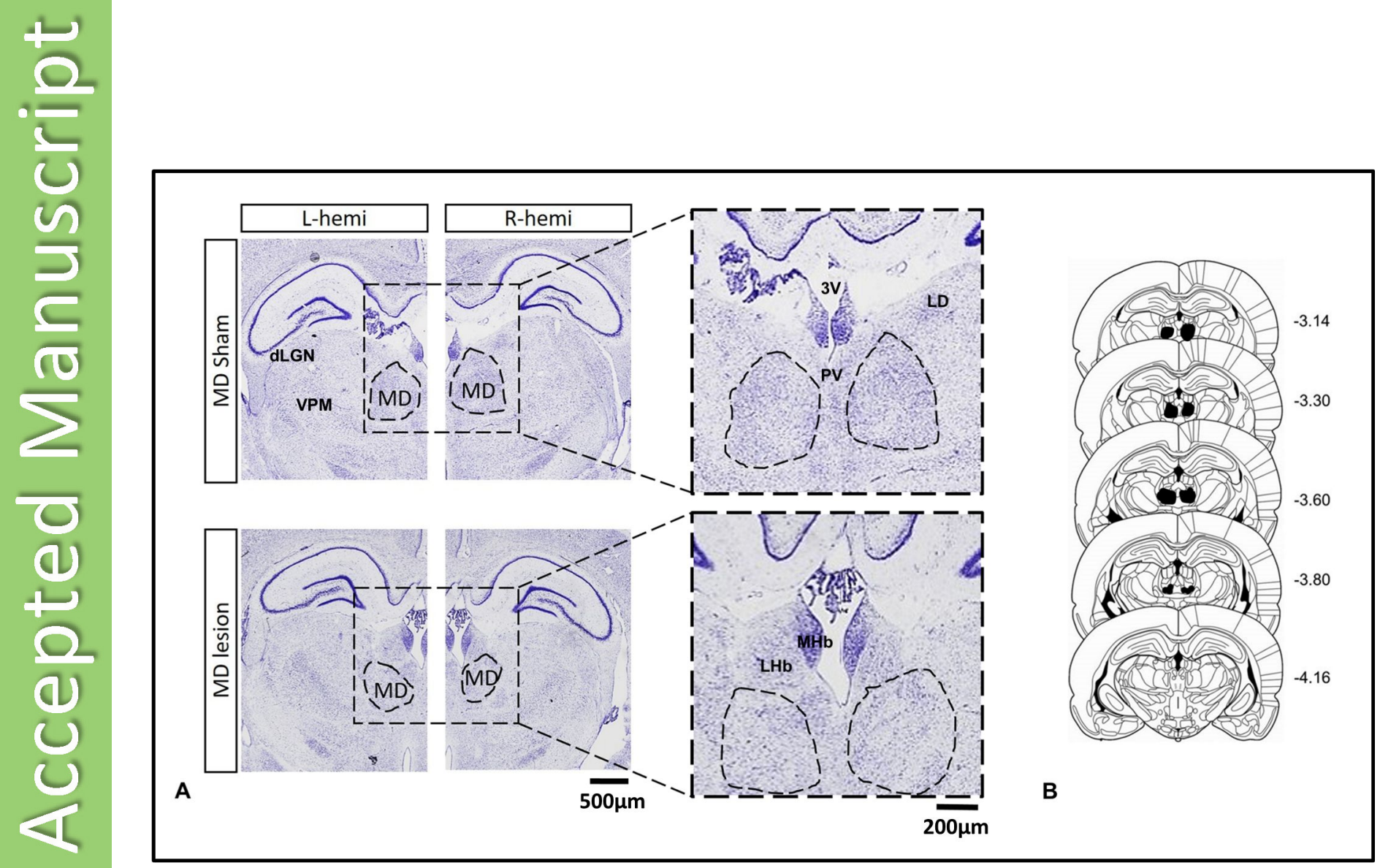

을 


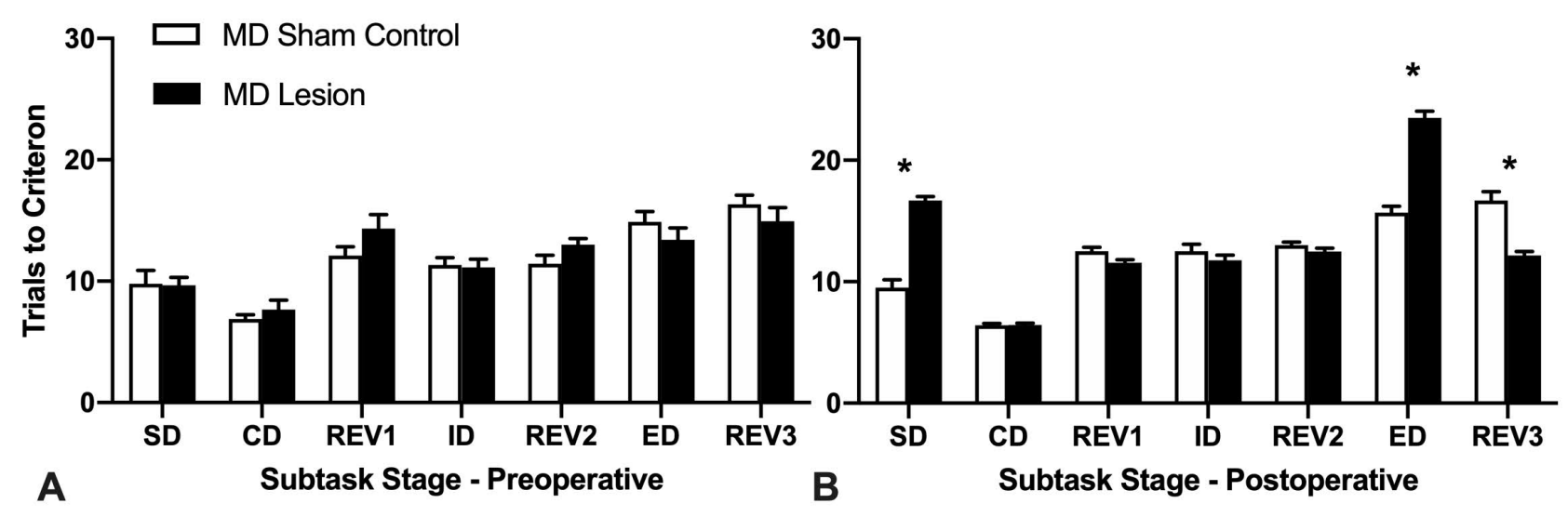



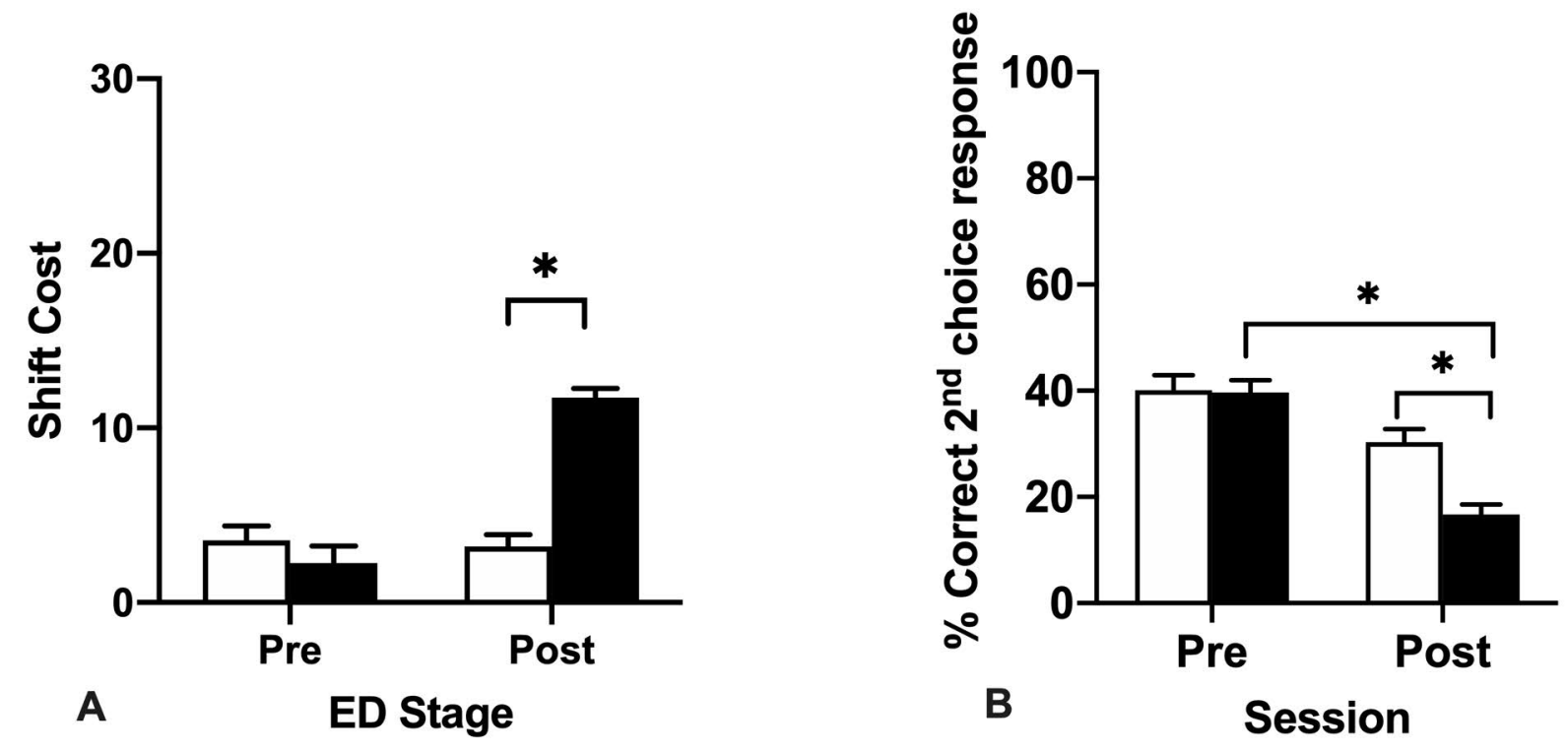
Correct trials

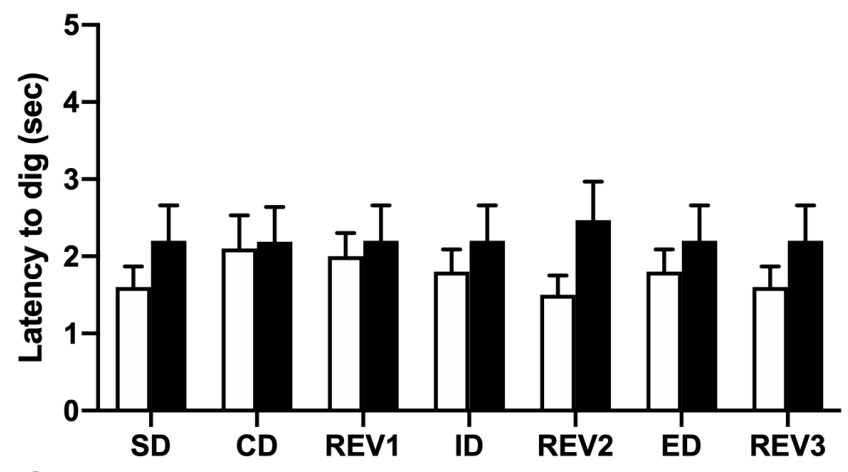

A

Subtask Stage - Preoperative

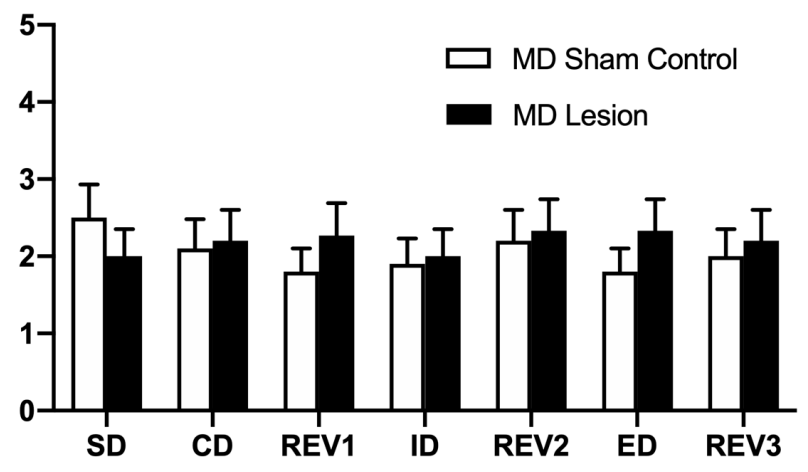

B
C Subtask Stage - Preoperative

\section{Error trials}

Subtask Stage - Postoperative

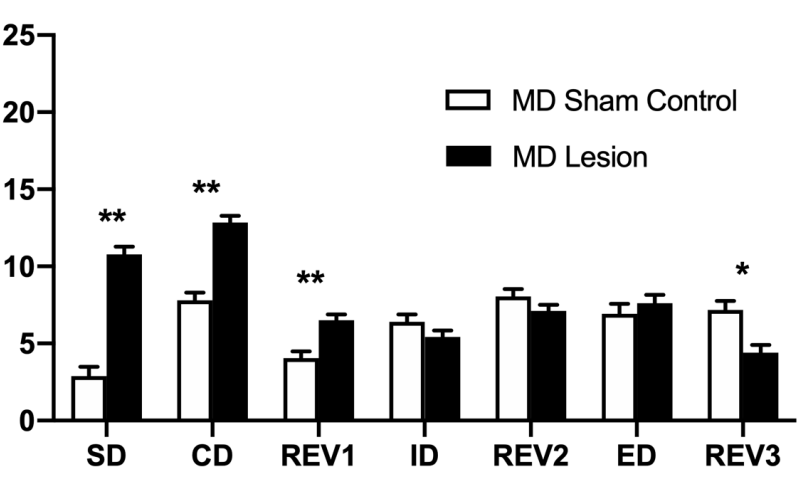

D 
Table 1. Sensory dimensions (odors and digging media) and stimulus features (O1-O6;

M1-M6) used in the different subtasks of the ID/ED attentional set-shifting task.

\begin{tabular}{|c|c|c|c|}
\hline \multicolumn{2}{|c|}{ Odors } & \multicolumn{2}{|c|}{ Digging media } \\
\hline O1 - Cinnamon & $\mathrm{O} 2$ - Ginger & M1 - Coarse tea & M2 - Fine tea \\
\hline O3 - Sage & O4 - Paprika & M3 - Sand & M4 - Grit \\
\hline O5 - Turmeric & O6 - Cloves & M5 - Coarse shavings & M6 - Fine shavings \\
\hline
\end{tabular}

